\title{
Existence of solutions of inverted variational inequalities
}

\section{SZILÁRD LÁSZLÓ}

\section{ABSTRACT.}

In this paper we introduce two new generalized variational inequalities and we give some existence results of the solutions for these variational inequalities involving operators belonging to a recently introduced class of operators. We show by examples, that our results fail outside of this class. Further, we establish a result that may be viewed as a generalization of Minty's theorem, that is, we show that under some circumstances the set of solutions of these variational inequalities coincide. We also show, the condition that the operators, involved in these variational inequalities, belong to the above mentioned class, is essential in obtaining this result. As application, we show that Brouwer's fixed point theorem is an easy consequence of our results.

BABEŞ-BOLYAI UNIVERSITY

FACULTY OF MATHEMATICS AND COMPUTER SCIENCE

M. KogĂLNiCEANU 1, 400084 Cluj-NAPOCA, Romania

E-mail address: laszlosziszi@yahoo.com 